\title{
First observation of elliptical sheet beam formation with an asymmetric solenoid lens
}

\author{
S. J. Russell, Z.-F. Wang, W. B. Haynes, R. M. Wheat, Jr., B. E. Carlsten, and L. M. Earley \\ Los Alamos National Laboratory, Los Alamos, New Mexico, USA \\ S. Humphries, Jr. \\ Field Precision, Albuquerque, New Mexico, USA \\ P. Ferguson \\ MDS Company, Oakland, California, USA \\ (Received 6 July 2005; published 18 August 2005)
}

\begin{abstract}
Currently ongoing at Los Alamos National Laboratory is a program to develop high-power, planar 100$300 \mathrm{GHz}$ traveling-wave tubes. A necessary part of this effort is a sheet electron beam source. Previously, we have described a novel asymmetric solenoid lens concept for transforming the circular beam from a high-perveance electron gun to a planar configuration. The lens is a standard electromagnetic solenoid with elliptical, instead of circular, pole apertures. The elliptical pole openings result in asymmetric focusing, which in turn forms an elliptical sheet beam suitable for our planar structures. Here we report the first experimental demonstration of this lens.
\end{abstract}

DOI: 10.1103/PhysRevSTAB.8.080401

PACS numbers: 84.40.Fe, 41.85.- $\mathrm{p}$, 41.75.Fr

\section{INTRODUCTION}

There is an emerging need for high-power (up to several kilowatts), high-frequency $(100-300 \mathrm{GHz})$, highbandwidth (up to 10\%) rf sources for advanced radar and communications. Previous work at Los Alamos National Laboratory has identified that sheet-beam driven travelingwave tubes (TWTs) can meet this need [1-3]. This type of rf source consists of a very thin electron beam passing through a planar, periodic slow-wave structure [1]. The promise of this technology is: (1) the rf structures lend themselves well to established microfabrication techniques; and (2) by spreading the electron beam in one dimension, we can transport a high net beam current, resulting in very high-power devices.

A key enabling technology for our rf source is an elliptical sheet-beam source. Specifically, we require a high aspect ratio elliptical electron beam with the nominal properties defined in Table I. The beam is wide in the horizontal $(x)$ direction and narrow in the vertical $(y)$ direction. There are two possible ways to construct this electron source: (1) by constructing a sheet-beam electron gun that directly produces a sheet beam; and (2) by transforming the beam from a conventional circular gun using a conversion element. We felt the latter approach provided a more flexible and potentially more useful beam source.

We took two approaches for our conversion element. The first transformed our circular beam using a quadrupole doublet magnetic lens [4]. Figure 1 is a typical image of an elliptical sheet beam formed using this method. Our second approach was to design and build an asymmetric solenoid lens [5].

The solenoid lens is simply a standard electromagnetic solenoid with elliptical, instead of circular, pole apertures
(Fig. 2). The asymmetric pole apertures lead to asymmetric focusing of the electron beam, resulting in the formation of an elliptical sheet beam downstream from the lens that is rotated approximately $40^{\circ}$ relative to the horizontal axis of the solenoid. We have simulated the lens performance using the three-dimensional (3D) magnetic field code, MAGNUM, and corresponding ray tracing code OMNITRAK $[5,6]$. In this paper we report the first experimental results of the lens' performance.

\section{DESCRIPTION OF EXPERIMENT}

A simple diagram showing the experimental layout is shown in Fig. 3. It consists of three main parts: the electron gun, the elliptical pole solenoid, and our imaging diagnostic. The electron gun and diagnostic exist inside the vacuum system (not shown), which typically operates in the low $10^{-8}$ Torr range. The solenoid is outside the vacuum system.

The electron gun has a standard Pierce geometry and was designed and built by MDS Company, Oakland, CA. It uses a conventional, dispenser type thermionic cathode from Semicon Associates that operates at $1070^{\circ} \mathrm{C}$. The gun was designed for pulsed operation at voltages up to $120 \mathrm{kV}$. Figure 4 is a description of the electron gun.

TABLE I. Nominal electron beam parameters for Los Alamos TWT program.

\begin{tabular}{lcc}
\hline \hline \multicolumn{1}{c}{ Quantity } & Symbol & Value \\
\hline Kinetic energy & $T_{e}$ & $120 \mathrm{keV}$ \\
Current & $I$ & $20 \mathrm{~A}$ \\
Full height of beam & $4 y_{\mathrm{rms}}$ & $0.5 \mathrm{~mm}$ \\
Full width of beam & $4 x_{\mathrm{rms}}$ & $10.0 \mathrm{~mm}$ \\
\hline \hline
\end{tabular}




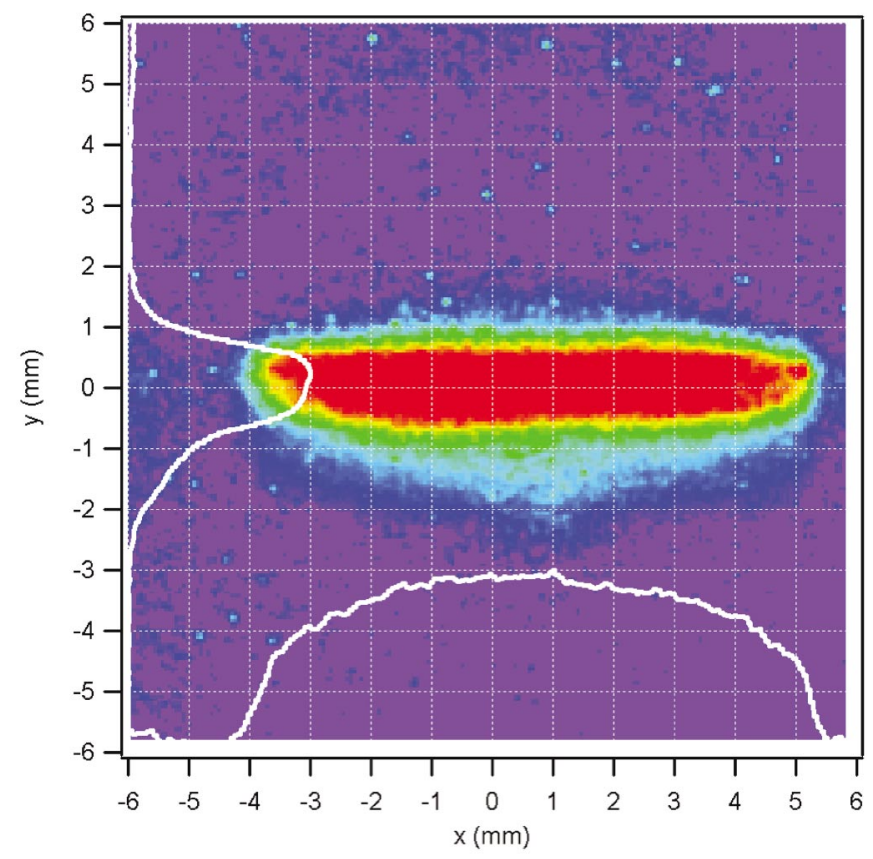

FIG. 1. Sheet electron beam image after circular beam is transformed using a quadrupole doublet magnetic lens. Beam energy was $43.5 \mathrm{kV}$ and beam current was $4.6 \mathrm{~A}$. (False color added during processing.)

The electron gun is powered by a solid state, insulated gate bipolar transistor (IGBT) modulator [7]. The modulator provides two electrical feeds to the electron gun. The first supplies a low voltage, ac current to the electron gun cathode heater. The second provides the high voltage pulse that operates the gun itself. Both modulator signals are output to a Dielectric Sciences high voltage (HV) cable rated for $160 \mathrm{kV}$. The $\mathrm{HV}$ cable is connected to the electron gun, which is under vacuum, via a Dielectric Sciences

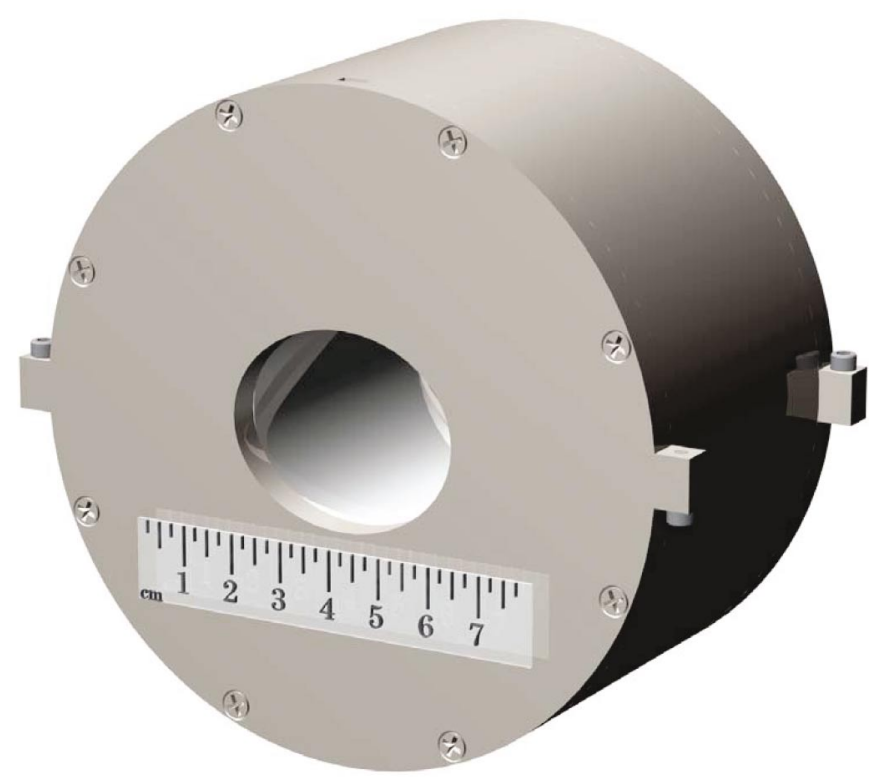

FIG. 2. Asymmetric solenoid lens with elliptical pole apertures in the steel yoke. The horizontal semiaxis radius of the apertures is 0.866 inches $(2.200 \mathrm{~cm})$ and the vertical semiaxis radius is 0.716 inches $(1.819 \mathrm{~cm})$. Both solenoid apertures are identical. The overall length of the lens is 2.75 inches $(6.985 \mathrm{~cm})$. The outer diameter of the solenoid yoke is 4.831 inches $(12.271 \mathrm{~cm})$.

ceramic insulated feedthrough [Fig. 4(a)]. The modulator is capable of producing voltage pulses up to $10 \mu \mathrm{s}$ in width at voltages between 10 and $120 \mathrm{kV}$. The pulse rise time on the electron gun is approximately $2 \mu \mathrm{s}$. A complete listing of the modulator operating parameters is given in Table II.

Downstream of the elliptical pole solenoid is our diagnostic, which is diagramed in Fig. 5. It consists of a circular 0.001-inch thick stainless steel foil followed by a circular 0.1-mm thick YAG:Ce scintillator crystal from MarkeTech
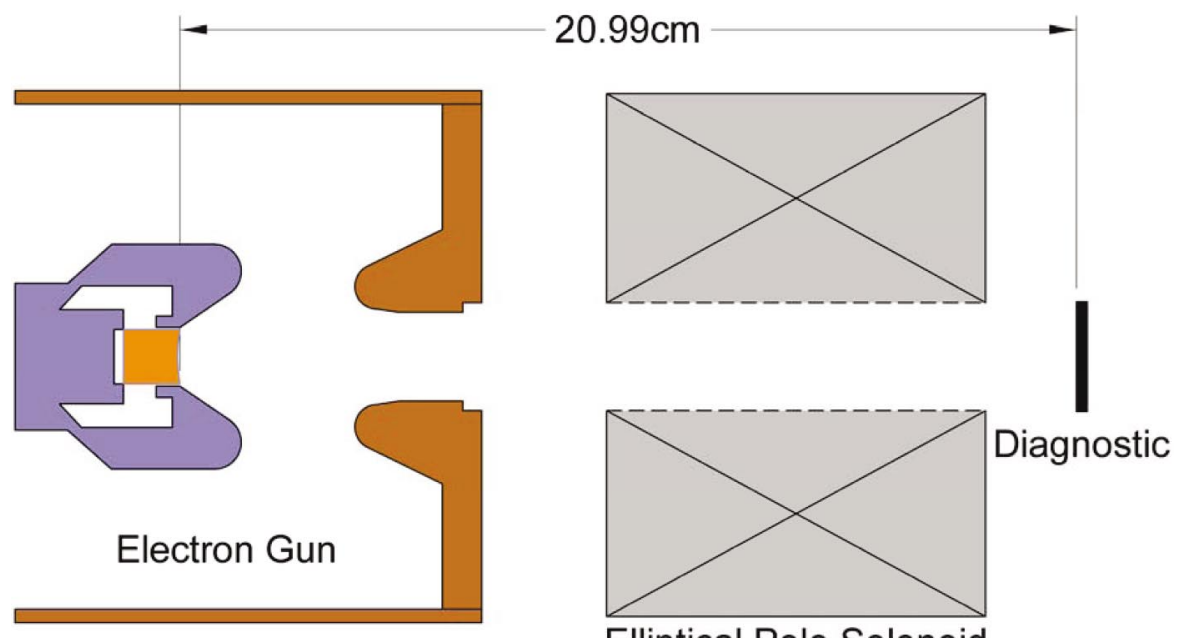

Elliptical Pole Solenoid

FIG. 3. Schematic of experimental layout. The electron gun and diagnostic are inside the vacuum system (not shown). The solenoid is outside the vacuum system. 

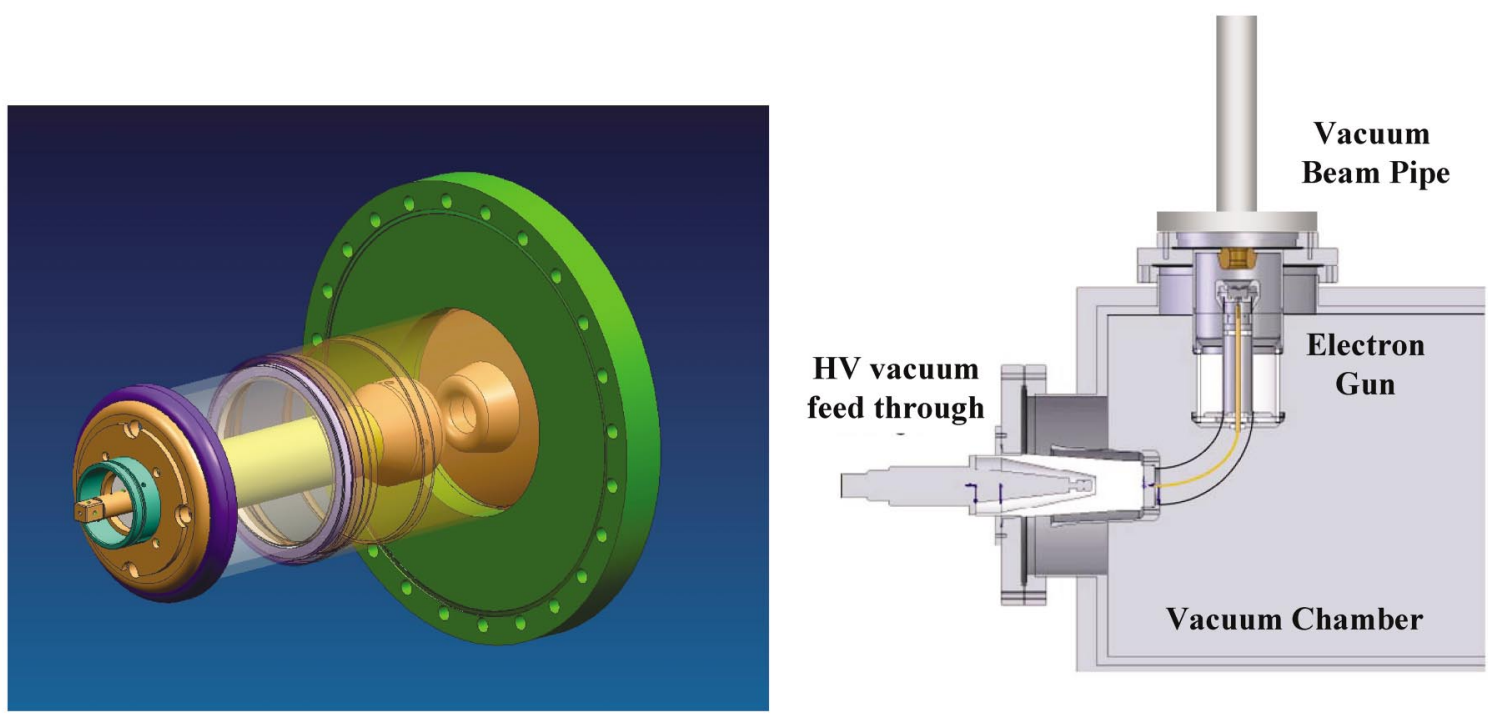

(A)

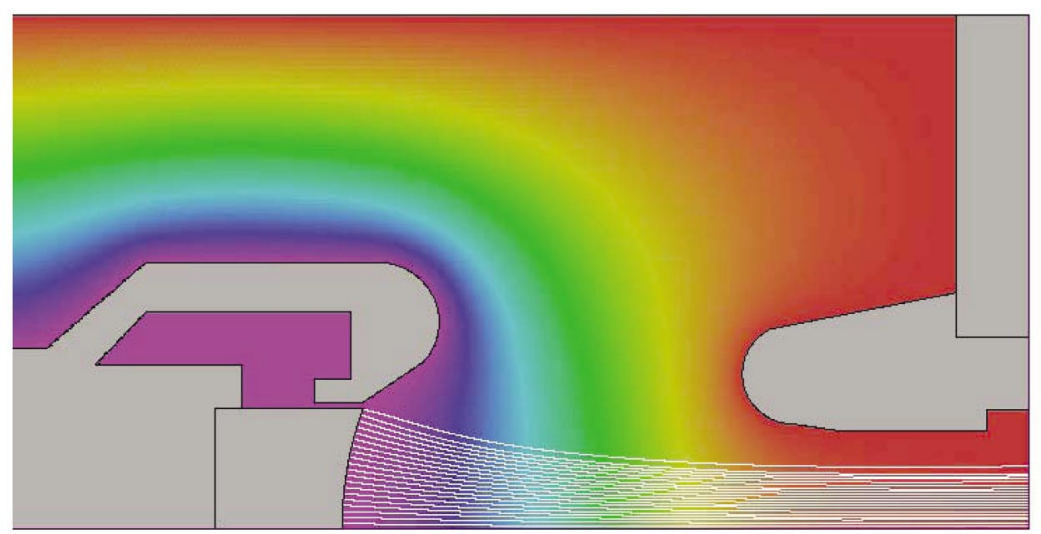

\section{Electric Potential Scale}

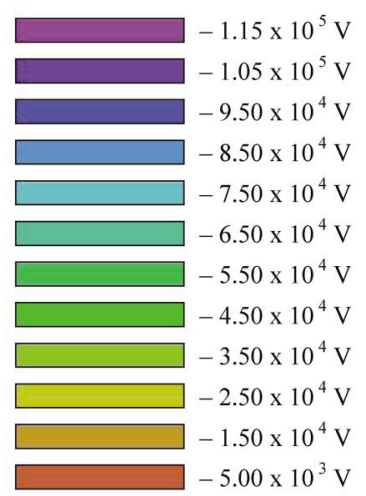

\section{Gun Parameters}

Perveance

$0.51 \mu$ Pervs

Beam radius at output

$0.6 \mathrm{~cm}$

Normalized beam emittance at output

$3.26 \mathrm{~mm} \mathrm{mrad}$

(B)

FIG. 4. Description of electron gun. (a) 3D CAD drawing of electron gun and drawing of gun mounted inside the vacuum chamber. Modulator connects to the electron gun via a high voltage cable to the HV vacuum feedthrough. (b) TRAK simulation of electron gun [6].

TABLE II. Modulator specifications.

\begin{tabular}{lc}
\hline \hline \multicolumn{1}{c}{ Quantity } & Value \\
\hline Voltage & $10-120 \mathrm{kV}$ \\
Load impedance at $120 \mathrm{kV}$ & $5200 \Omega$ \\
Pulse rise time & $\sim 2 \mu \mathrm{s}$ \\
Pulse width & $4-10 \mu \mathrm{s}$ \\
Repetition rate & Single pulse $-10 \mathrm{~Hz}, 1 \mathrm{~Hz}$ nominal \\
Electron gun filament & $8 \mathrm{~V}, 7.5 \mathrm{~A} \mathrm{(AC)}$ \\
\hline \hline
\end{tabular}




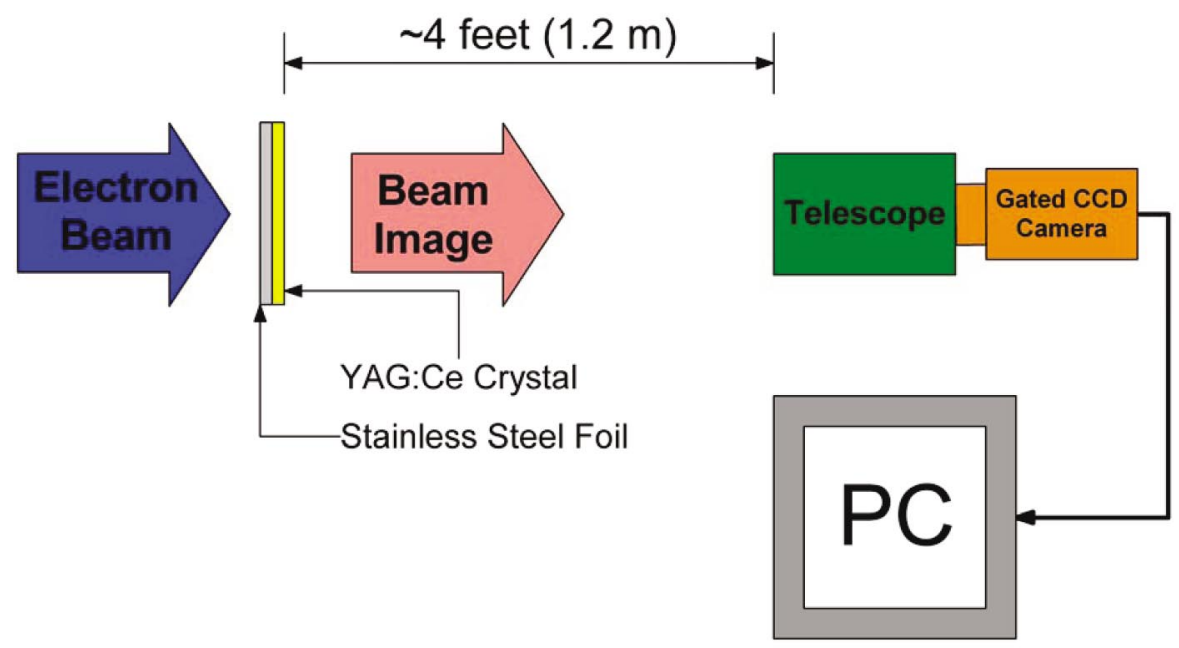

FIG. 5. Diagram of beam imaging diagnostic.

International. The active area of this detector is 0.84 inches in diameter and it is located $21 \mathrm{~cm}$ downstream from the electron gun cathode (Fig. 3). The steel foil stops the electron beam, generating a pulse of Bremsstrahlung $\mathrm{x}$ rays. In turn, the $\mathrm{x}$ rays are imaged by the scintillator crystal, which has a decay constant of 70 ns. A Questar telescope focuses this image into an electronically gated, intensified CCD camera from Xybion Electronic Systems Corporation (model ISG 240). We set the gated camera to capture a small time slice of the beam signal (typically $0.5 \mu \mathrm{s}$ ). In turn, a Spiricon ${ }^{\circledR} 12$ bit frame grabber (model LBA-500PC) residing in a PC running Spiricon ${ }^{\circledR}$ PC Laser Beam Analyzer software captures the camera output.

The optical resolution of our diagnostic (camera plus telescope) is approximately $30 \mu \mathrm{m}$. However, the resolution we achieve when we convert the electron beam to an $\mathrm{x}$ ray signal is harder to quantify. The steel foil is needed because the YAG:Ce crystal by itself cannot withstand the beam power; however, the resulting $\mathrm{x}$ rays are scattered by both the foil and the crystal, causing the image to blur. We are currently analyzing the diagnostic using the radiation transport code GAMBET [6]. Early results indicate a resolution of about $0.1 \mathrm{~mm}$, approximately the thickness of the foil plus the crystal $(0.13 \mathrm{~mm})$.

In the experiment reported here, we operated the electron gun at a relatively low voltage $(\sim 20 \mathrm{kV})$. We then varied the current of the elliptical pole solenoid and recorded the beam image at the diagnostic position. We operated at this low voltage for two reasons: (1) to avoid damage to our diagnostic and (2) to mitigate a background gas ionization focusing effect that interferes with our results. This second effect will be discussed further in the next section.

Figure 6 is a plot of a typical voltage pulse versus time from our experiment. We show in Fig. 6 the voltage output from the modulator, the beam current, and the location and width of the camera gate signal. This plot shows that by using a gated camera we can effectively eliminate the long rise time of the modulator and sample the beam at a single energy.

Our voltage diagnostic is located at the modulator transformer secondary, prior to the high voltage cable and vacuum feedthrough that connect the modulator and electron gun. It does not directly measure the voltage across the electron gun gap, introducing ambiguity into our measurement of the actual electron beam energy. The measurement of the electron beam current and the camera gate signal are both much more accurate.

\section{EXPERIMENTAL RESULTS AND ANALYSIS}

Figure 7 shows a series of beam images as we increase the solenoid current and focus the beam through a waist in the vertical direction. The top images are from experiment and the lower images are from MAGNUM/OMNITRAK simulations [6]. In Fig. 8, we plot the horizontal and vertical root-mean-square (rms) widths of the beam versus the solenoid current, along with simulation results for comparison. The minimum beam width in the vertical dimension occurred at a solenoid current of $4.3 \mathrm{~A}$ (peak magnetic field $247 \mathrm{G}$ ). The measured beam dimensions at this point are

$$
\begin{aligned}
& x_{\mathrm{rms}}=2.1 \mathrm{~mm} \Rightarrow x_{\mathrm{width}}=4 x_{\mathrm{rms}}=8.4 \mathrm{~mm}, \\
& y_{\mathrm{rms}}=0.4 \mathrm{~mm} \Rightarrow y_{\text {width }}=4 y_{\mathrm{rms}}=1.6 \mathrm{~mm} .
\end{aligned}
$$

Some observations of the data are the following: (1) the measured images in Fig. 7 show a beam asymmetry, especially in Fig. 7(a), (2) the measured images show a "hot spot" in the beam center that does not appear in the simulations, (3) the resulting elliptical beam has a larger vertical waist than predicted by the simulations, and (4) the beam proceeds through a double vertical minimum as the solenoid current is increased (Fig. 8). We will discuss each point in turn. 


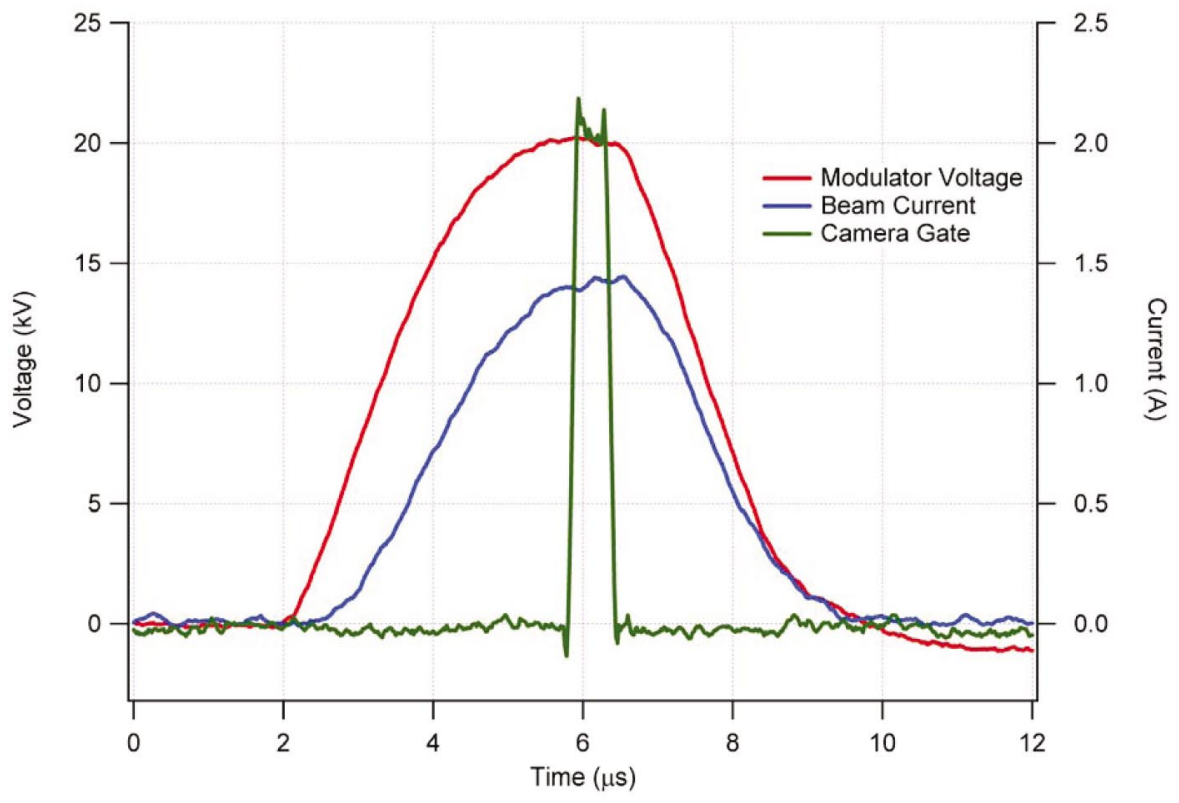

FIG. 6. Typical voltage pulse from our experiment. We show the voltage pulse from the modulator (red), the beam current (blue), and the camera gate signal (green).

The asymmetry of the measured beam images is easily explained. Figure 9 shows the electron beam directly out of the electron gun before it enters the elliptical pole solenoid lens. It shows a clearly asymmetric beam. Upon investiga-

Solenoid Current 3.9 A
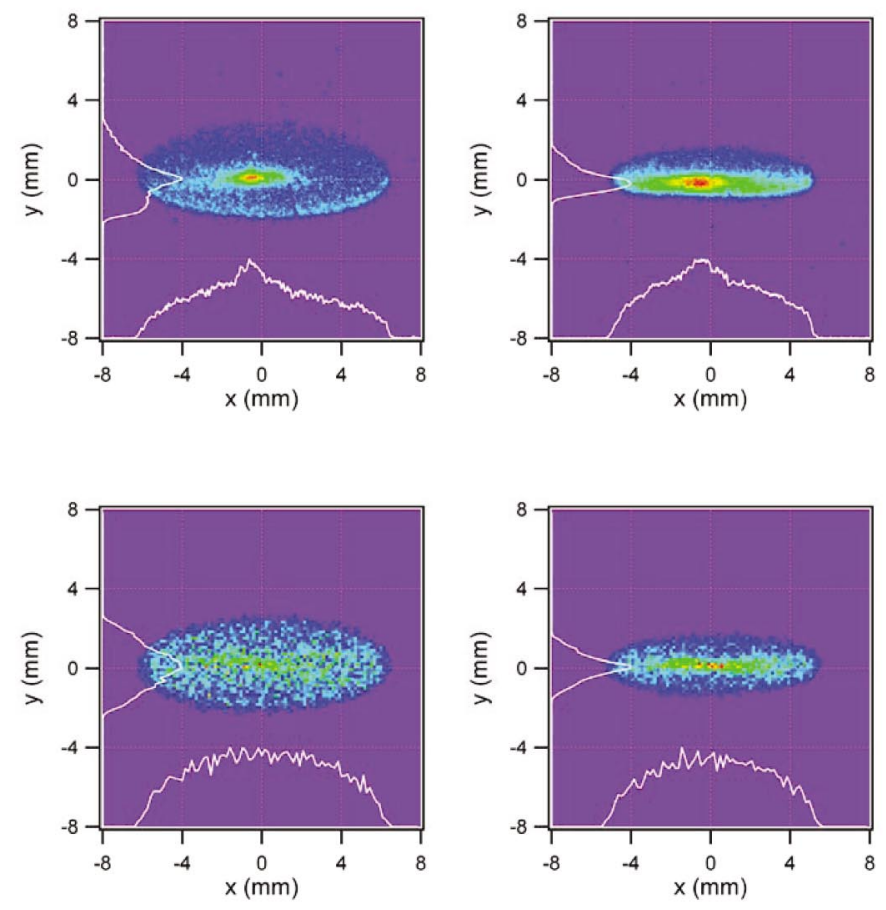

(A)

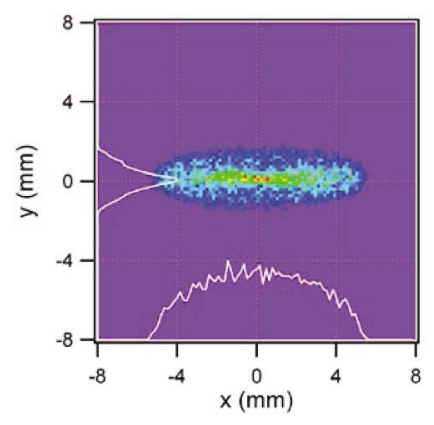

(B)

tion, we discovered the gun's cathode was not properly centered in the gun structure during the experiment.

We postulate that the hot center point in the measured images is caused by a time and energy dependent ioniza-

Solenoid Current 4.3 A
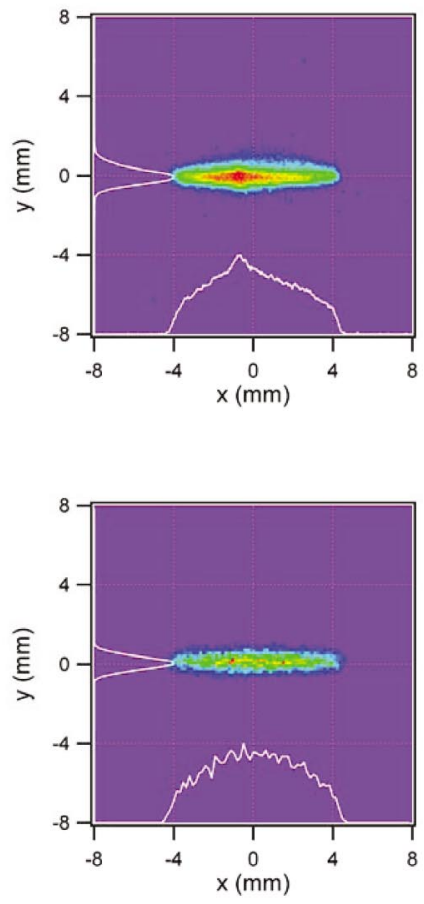

(C)
Solenoid Current 4.5 A
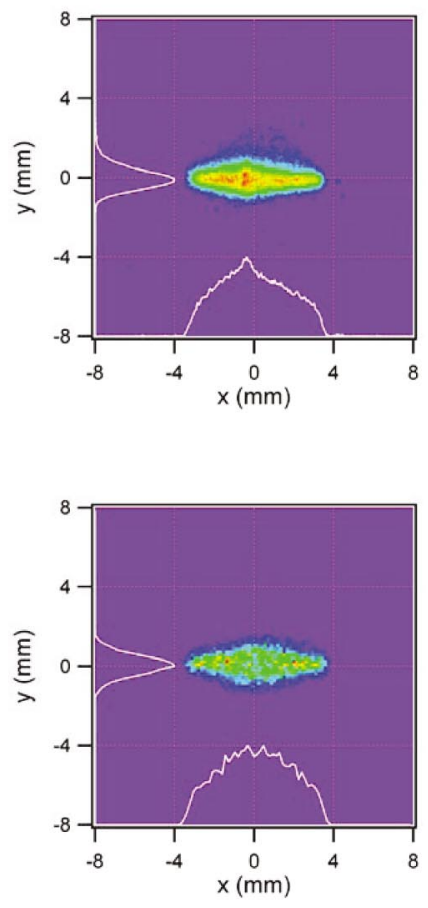

(D)

FIG. 7. Beam images from experiment (top) compared to images generated from MAGNUM/OMNITRAK simulations (bottom). False color is added for better contrast. (a) Solenoid current 3.9 A (peak magnetic field 224 G). (b) Solenoid current 4.1 A (peak magnetic field 236 G). (c) Solenoid current 4.3 A (peak magnetic field 247 G). (d) Solenoid current 4.5 A (peak magnetic field 259 G). 


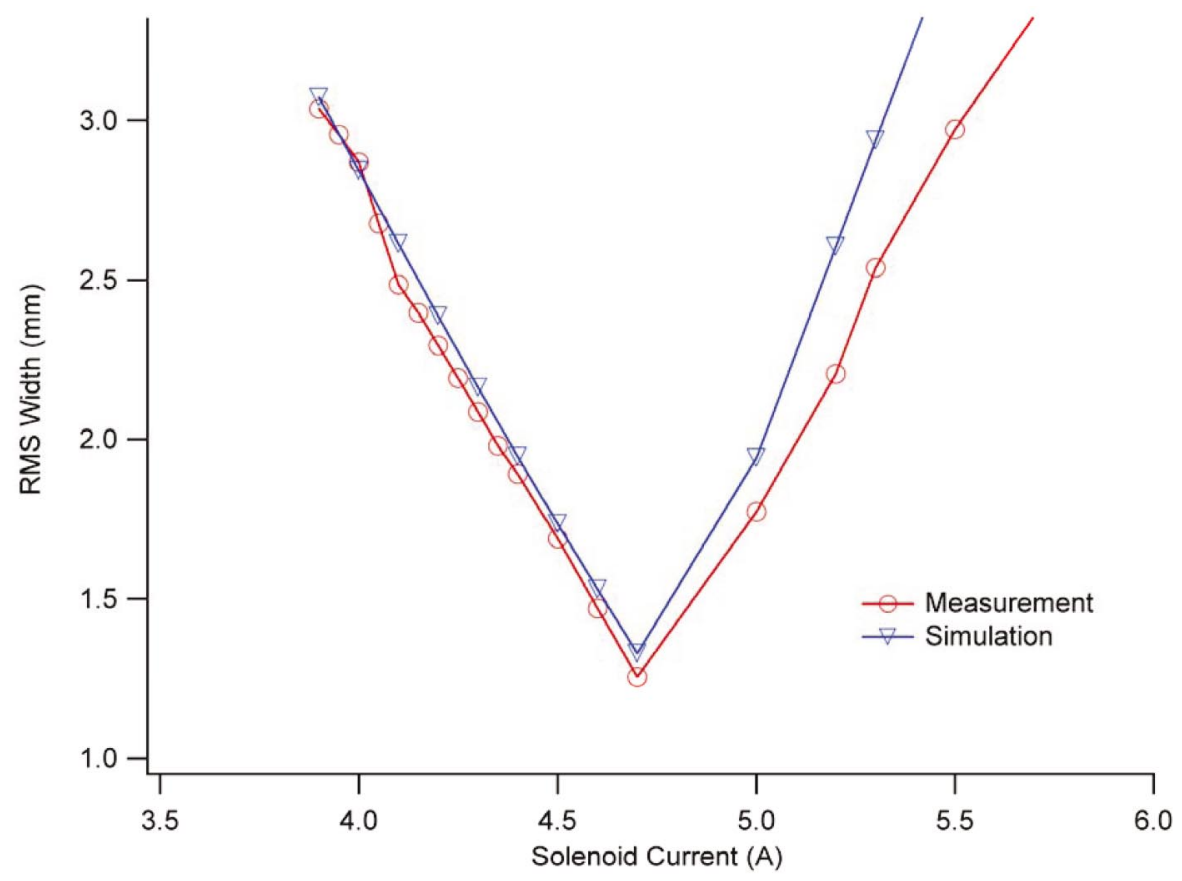

(A)

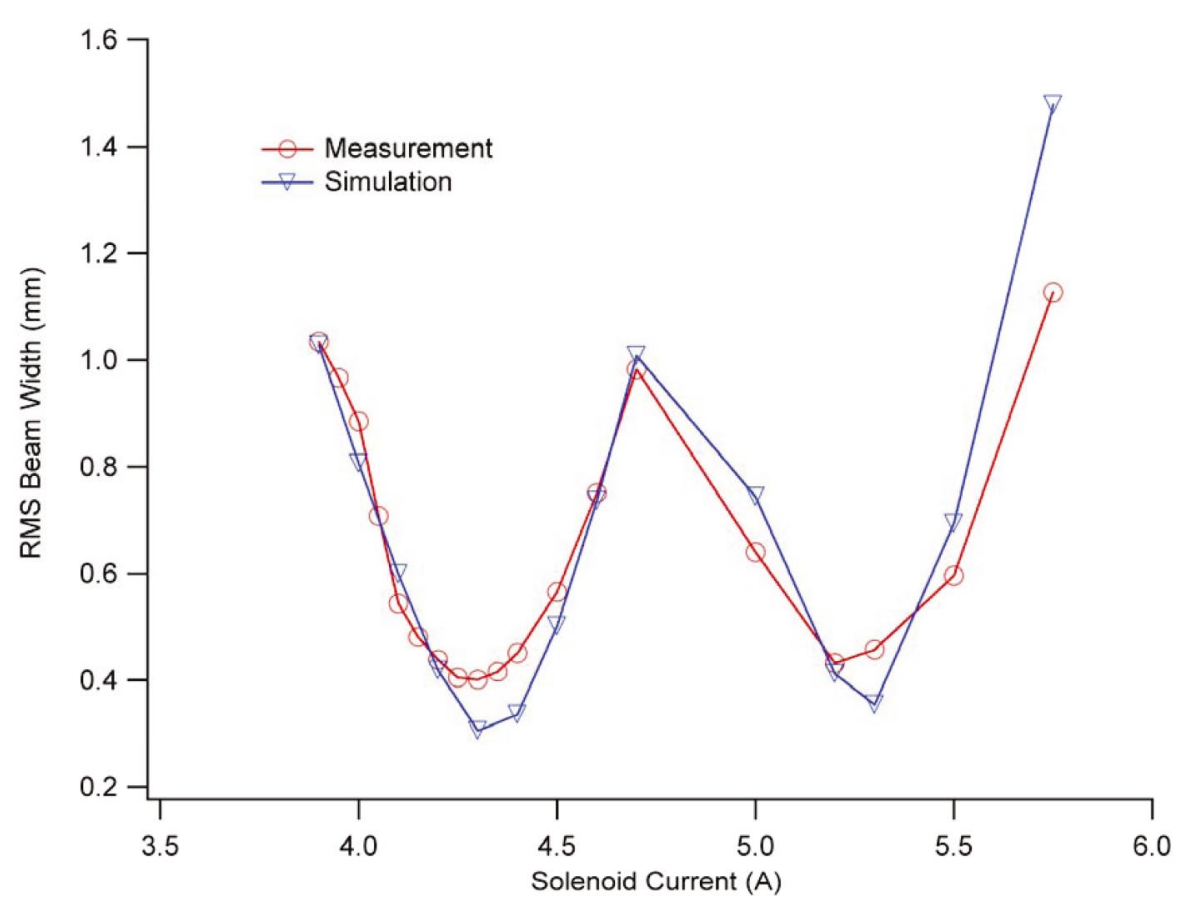

(B)

FIG. 8. rms beam width versus solenoid current from measurement and simulation. (a) Horizontal rms width. (b) Vertical rms width.

tion effect. When the beam strikes the stainless steel foil in the diagnostic, contaminates (such as monolayers of residual gas which condense on the foil) are liberated from the foil surface. This creates a spike in the local background gas pressure. This background gas is ionized by electrons early in the beam, resulting in a time dependent, distributed focusing force that acts on electrons later in the beam pulse. If this effect is indeed the cause, we should expect that it would be exacerbated when we increase the gun voltage (higher beam current and energy) and when we 


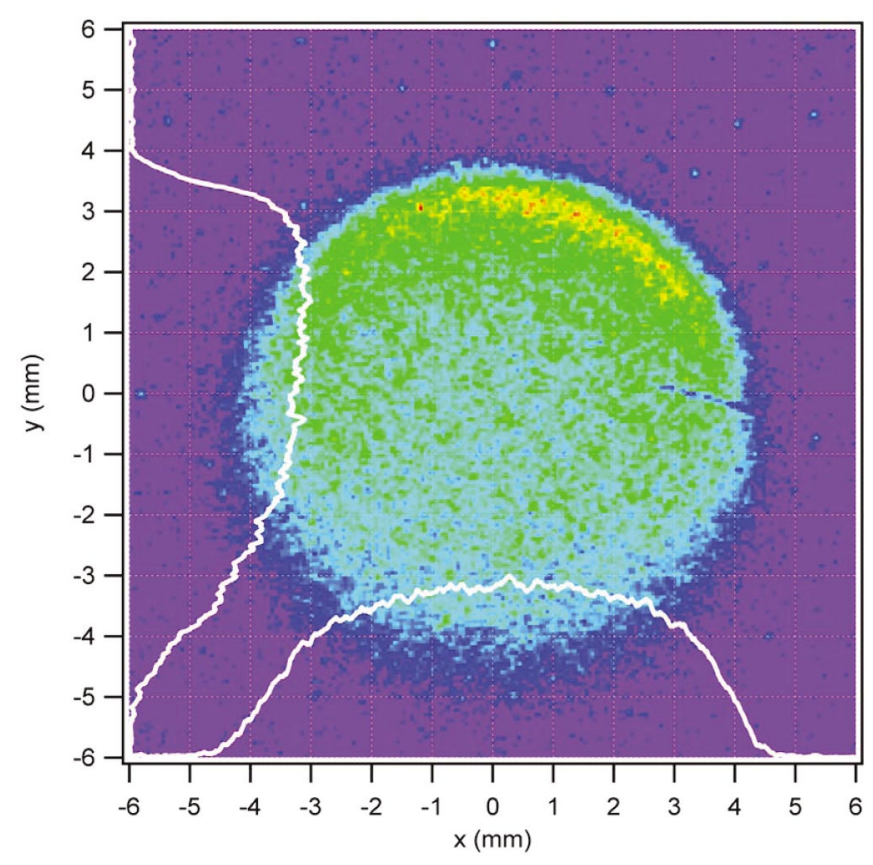

FIG. 9. Image of electron beam directly out of electron gun showing beam asymmetry due to misaligned cathode.

move the camera gate later in the beam pulse. In fact, preliminary investigation shows that these effects are indeed true.

The simulations show reasonable agreement with the measurements. However, they also predict a smaller vertical beam size than what was actually measured (Figs. 7 and 8). This discrepancy has more than one possible explanation. First, as mentioned in the last section, our measurement of the gun voltage is ambiguous. To accurately predict the beam energy, we make the assumption that the electron gun is operating in a space charge limited mode. Then, the beam current is related to the gap voltage by the gun perveance [8]:

$$
I_{\text {beam }}=P_{\text {gun }} V_{\text {gun }}^{3 / 2}
$$

The gun perveance, $P$, is a constant determined by the electron gun geometry, which we know from simulation (Fig. 4) and have confirmed by measurement. With a beam current of 1.42 A (Fig. 6) during the camera gate, we can infer the gun voltage was $19.7 \mathrm{kV}$. However, the agreement between measurements and simulations is better when we assume in the model an electron gun voltage of $21 \mathrm{kV}$, while maintaining a 1.42 beam current. This tends to indicate that our assumption that the gun is operating in a space charge limited mode is incorrect. However, other effects that could contribute to the discrepancy are the distributed ionization focusing effect, which is not present in the simulation, or the misaligned cathode, which is also absent from the model. Another possibility that could contribute to the discrepancy is the resolution of the diagnostic.
A critical beam property that could also explain the disagreement between the predicted vertical beam size and the measured beam size is the beam emittance. Consider the horizontal and vertical rms envelope equations as the electron beam propagates in the longitudinal (z) direction [8]:

$$
\begin{gathered}
\frac{d^{2} X}{d z^{2}}+k_{x}(z) X-\frac{K}{2(X+Y)}-\frac{\varepsilon_{x}^{2}}{X^{3}}=0, \\
\frac{d^{2} Y}{d z^{2}}+k_{y}(z) Y-\frac{K}{2(X+Y)}-\frac{\varepsilon_{y}^{2}}{Y^{3}}=0, \\
X \equiv\left\langle x^{2}\right\rangle, \\
Y \equiv\left\langle y^{2}\right\rangle,
\end{gathered}
$$

where the angled brackets indicate an rms average. The terms $k_{x}(z)$ and $k_{y}(z)$ represent the linear focusing terms in the two planes. The space charge force is proportional to the generalized perveance,

$$
K=\frac{q_{e} I_{\text {beam }}}{2 \pi \varepsilon_{0} m_{e} \gamma^{3} v_{\text {beam }}^{3}},
$$

where $q_{e}$ is the electron charge, $I_{\text {beam }}$ is the electron beam current, $m_{e}$ is the electron mass, $\gamma$ is the standard relativistic parameter, and $v_{\text {beam }}$ is the beam velocity. The final term is the emittance term, which is analogous to a repulsive pressure force acting on the rms envelope. The rms, unnormalized emittances are defined as

$$
\begin{aligned}
& \varepsilon_{x}=\sqrt{\left\langle x^{2}\right\rangle\left\langle\left(\frac{d x}{d z}\right)^{2}\right\rangle-\left\langle x \frac{d x}{d z}\right\rangle^{2}}, \\
& \varepsilon_{y}=\sqrt{\left\langle y^{2}\right\rangle\left\langle\left(\frac{d y}{d z}\right)^{2}\right\rangle-\left\langle y \frac{d y}{d z}\right\rangle^{2}} .
\end{aligned}
$$

We see in Eqs. (4) and (5) that, near a beam waist, the emittance term can be quite large. For our beam, we expect the vertical emittance term to be on the order of 100 times larger than the space charge term at the vertical waist. Therefore, small differences in the emittance predicted by the model and the actual beam emittance in the experiment can result in significant discrepancies between measurement and simulation. In particular, a larger emittance in the experiment would result in a larger observed vertical waist.

Finally, we would like to draw attention to the double vertical minimum indicated in Fig. 8. As the solenoid current is increased, we see the first vertical minimum occurring at $4.3 \mathrm{~A}$. A second minimum occurs at $5.2 \mathrm{~A}$. In the second minimum, the beam actually passes through a focus inside the solenoid and then is refocused at the diagnostic position. We find it somewhat remarkable that the beam quality is in large part preserved to this second 
minimum, achieving almost the same vertical size as the first vertical waist.

\section{SUMMARY AND CONCLUSIONS}

We have successfully tested our asymmetric solenoid lens. We have measured a high aspect ratio sheet beam and measurement agreed reasonably well with simulations. Despite uncertainties in absolute operating conditions, we have demonstrated that this type of lens is a viable way to transform circular electron beams to high quality elliptical beams that are suitable for our planar, microfabricated TWT amplifiers.

\section{ACKNOWLEDGMENTS}

The authors would like to acknowledge Richard Brown, Randolf Carlson, Michael V. Fazio, Frank P. Romero, and Floyd E. Sigler for their contributions to the work presented here.
[1] B. E. Carlsten, S. J. Russell, L. M. Earley, F. L. Krawczyk, J.M. Potter, P. Ferguson, and S. Humphries, Jr., IEEE Trans. Plasma Sci. 33, 85 (2005).

[2] B. E. Carlsten, Phys. Plasmas 8, 4585 (2001).

[3] B. E. Carlsten, Phys. Plasmas, 9, 5088 (2002).

[4] M. A. Basten and J. H. Booske, IEEE Trans. Plasma Sci. 22, 960 (1994).

[5] S. Humphries, S. Russell, B. Carlsten, L. Earley, and P. Ferguson, Phys. Rev. ST Accel. Beams 7, 060401 (2004).

[6] GAMBET, MAGNUM, OMNITRAK, and TRAK software, copyright Field Precision, Albuquerque, NM.

[7] L. M. Earley, R.W. Brown, R. L. Carlson, P. Ferguson, W. B. Haynes, H. C. Kirbie, S. J. Russell, F. E. Sigler, E. I. Smirnova, and R. M. Wheat, Jr., in Proceedings of the 26th International Power Modulator Symposium and 2004 High Voltage Workshop, San Francisco, CA (IEEE, New York, 2004), p. 285.

[8] See, for instance, M. Reiser, Theory and Design of Charged Particle Beams (John Wiley \& Sons, New York, NY, 1994). 